\title{
Two-Photon Polymerization of Albumin Hydrogel Nanowires Strengthened with Graphene Oxide
}

\author{
Nikita Nekrasov $^{1}{ }^{\mathbb{D}}$, Natalya Yakunina ${ }^{1}$, Vladimir Nevolin ${ }^{1}$, Ivan Bobrinetskiy ${ }^{1,2} \mathbb{D}$, Pavel Vasilevsky $^{3}$ \\ and Alexander Yu. Gerasimenko $3,4, * \mathbb{D}$
}

1 Center for Probe Microscopy and Nanotechnology, National Research University of Electronic Technology, 124498 Moscow, Russia; 8141147@gmail.com (N.N.); natali.swan.1999@mail.ru (N.Y.); vkn@miee.ru (V.N.); bobrinet@biosense.rs (I.B.)

2 BioSense Institute-Research and Development Institute for Information Technologies in Biosystems, University of Novi Sad, 21000 Novi Sad, Serbia

3 Institute of Biomedical Systems, National Research University of Electronic Technology, 124498 Moscow, Russia; pavelvasilevs@yandex.ru

4 Institute for Bionic Technologies and Engineering, I.M. Sechenov First Moscow State Medical University, Bolshaya Pirogovskaya Street 2-4, 119991 Moscow, Russia

* Correspondence: gerasimenko@bms.zone; Tel.: +7-926-702-9778

Citation: Nekrasov, N.; Yakunina, N.; Nevolin, V.; Bobrinetskiy, I.; Vasilevsky, P.; Gerasimenko, A.Y. Two-Photon Polymerization of Albumin Hydrogel Nanowires Strengthened with Graphene Oxide. Biomimetics 2021, 6, 66 .

https://doi.org/10.3390/

biomimetics 6040066

Academic Editor: Hermann Ehrlich

Received: 4 November 2021

Accepted: 22 November 2021

Published: 24 November 2021

Publisher's Note: MDPI stays neutral with regard to jurisdictional claims in published maps and institutional affiliations.

Copyright: (c) 2021 by the authors. Licensee MDPI, Basel, Switzerland. This article is an open access article distributed under the terms and conditions of the Creative Commons Attribution (CC BY) license (https:// creativecommons.org/licenses/by/ $4.0 /)$.

\begin{abstract}
Multifunctional biomaterials can pave a way to novel types of micro- and nanoelectromechanical systems providing benefits in mimicking of biological functions in implantable, wearable structures. The production of biocomposites that hold both superior electrical and mechanical properties is still a challenging task. In this study, we aim to fabricate 3D printed hydrogel from a biocomposite of bovine serum albumin with graphene oxide (BSA@GO) using femtosecond laser processing. We have developed the method for functional BSA@GO composite nanostructuring based on both two-photon polymerization of nanofilaments and direct laser writing. The atomicforce microscopy was used to probe local electrical and mechanical properties of hydrogel BSA@GO nanowires. The improved local mechanical properties demonstrate synergistic effect in interaction of femtosecond laser pulses and novel composite structure.
\end{abstract}

Keywords: two-photon polymerization; bovine serum albumin; graphene oxide; filament propagation; nanowires; mechanical properties; electrical properties

\section{Introduction}

Bio-inspired nanoelectromechanical devices (NEMS) are actively rising technology in advanced functional systems paving the way for modern technology of soft, stretchable, self-healing next-generation systems [1,2]. Using nanocarbon materials like carbon nanotubes (CNT) and graphene in complex composites improves its electro-active and scaffold properties. Recently these materials were successfully implemented in the development of $\mathrm{pH}$ sensitive hydrogels where swelling rate is dependent either on alkaline or acid environments [3,4].

Albumin is a protein that finds its application in different areas of bionanohybrids production and state-of-the-art nanotechnology [5]. Albumin is widely applied to laserwelded biological tissues as a laser solder [6]. Polymerization of bovine serum albumin (BSA) molecules is possible either by direct single-photon processing under UV irradiation [7], thermal treatment [8], or two-photon polymerization (TPP) [9,10]. The later process requires a photoinitiator (PI) [11]. Two-photon polymerization of BSA is actively employed to develop devices, such as microactuators [12] and optical nanodevices [9,13]. BSA-based microdevices have demonstrated a high environmental $\mathrm{pH}$ sensitivity, that makes BSA composites ideal materials for stimuli-sensitive biocompatible applications [9]. Femtosecond laser programmed artificial musculoskeletal system was proposed for soft smart muscle based on pH-responsive BSA [10]. 
Previously we demonstrated that CNT/BSA composites with varied conductivity and concentration can be used as biocompatible materials for sensor application [14] or scaffold for cells growth [15]. The 2D materials, such as graphene, provide advanced properties of composites for lower load concentration while maintaining flexibility and stretchable properties. Different methods were suggested for development of graphene/albumin aggregates, either by hydrophobic interactions [16], Van der Waals [17], or chemical crosslinking $[18,19]$. The graphene in form of graphene oxide (GO) is actively used in composites formation due to its hydrophilic properties, while for conductive properties the subsequent reduction is needed. The reduction of GO by standard chemical methods was suggested in the process of mixing with hydrogel materials [3]. In addition, the effect of ionic strength on electrostatic interaction of albumin with GO results in albumin binding energy modulation and increase of protein absorption density for higher ionic strength of solutions [20]. Nevertheless, the development of nanostructured functional composites based on proteins and graphene is still a challenging task.

In this work, we suggest the use of femtosecond pulsed laser for hydrogel nanowires formation using two-photon polymerization of albumin and graphene oxide composites. The local electrical and mechanical properties of BSA and hydrogel nanowires were investigated. The improvement of mechanical properties for BSA@GO hydrogel nanowires was demonstrated even at low GO concentration.

\section{Materials and Methods}

BSA powder was purchased from BioClot (Aidenbach, Germany). GO was provided by Smagulova S.A. (M.K. Ammosov North-Eastern Federal University, Yakutsk, Russia) in $4.7 \mathrm{~g} / \mathrm{L}$ water solution. Photoinitiator (methylene blue (MB), 1\% water solution) was purchased from LLC Zoomir (Moscow, Russia).

Two-photon polymerization was performed on tunable Ti:Sapphire femtosecond laser Chameleon (Coherent, Santa Clara, CA, USA) with wavelength set to $715 \mathrm{~nm}$ with $140 \mathrm{fs}$ pulse duration, $80 \mathrm{MHz}$ repetition rate, and varied power up to $30 \mathrm{~mW}$. To tune the power of the laser radiation, we used a motorized optical attenuator OAGP-M based on Glan prism (Avesta, Moscow, Russia). The initial beam had a Gaussian profile (Figure 1). The beam diameter at the $1 / \mathrm{e}^{2}$ level was $1.2 \pm 0.1 \mathrm{~mm}$. The images of the spatial profile of the laser beam were obtained using an SP620U CCD camera (Ophir Optronics Solutions, Jerusalem, Israel). The pulse repetition rate was controlled using an MSO6054A oscilloscope (Agilent Technologies, Santa Clara, CA, USA). Samples were placed on the motorized XY 8MTF scanning stage (Standa, Vilnius, Lithuania). The laser pulses reached the sample surface through an optical upright microscope with $60 \times$ objective $(\mathrm{NA}=0.65)$. The accumulated energy for TPP at average pulse power varied via the scanning speed of XY stage. A SVETOLIT-50 mercury lamp (Svetolit, Moscow, Russia) was used for UV experiments.
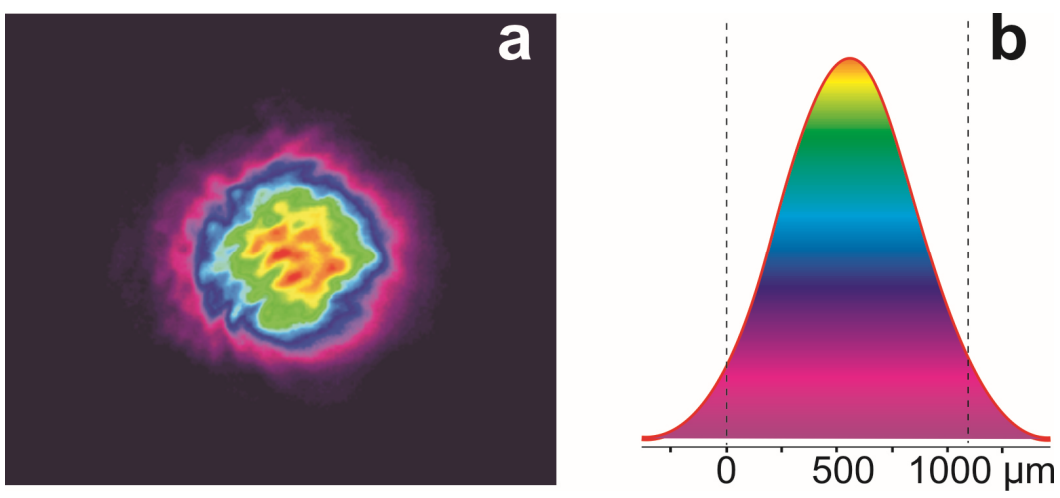

Figure 1. 2D beam profile visualization (a) and Gaussian shape of the initial beam (b) of femtosecond laser. 
BSA was dissolved in $1 \times$ PBS (phosphate buffer saline) $(\mathrm{pH}=7.4)$ in the concentration of $0.6 \mathrm{~g} / \mathrm{mL}$. To avoid the fast aggregation of proteins, we added $100 \mathrm{mg}$ of BSA in $1 \mathrm{~mL}$ of PBS followed by ultrasonic treatment for $5 \mathrm{~min}$ after each step. MB was added to BSA solution to reach the $16 \mathrm{mM}$ concentration of $\mathrm{MB}$. One hour of ultrasonic treatment in cold water was used to prevent the denaturation of proteins. To produce BSA@GO solution, $10 \mu \mathrm{L}$ of GO solution was added to $1 \mathrm{~mL}$ of PBS prior to BSA to reach $0.005 \%$ concentration of graphene.

BSA hydrogel nanowires were characterized via atomic force microscopy (AFM, Solver Pro (NT-MDT, Moscow, Russia)). Two types of silicon cantilevers were used: standard silicon cantilevers with resonance frequency $\mathrm{F}_{\text {res }}=200 \mathrm{kHz}$ (NSG01, TipsNano, Tallin, Estonia) for force-distance curves and with $35 \mathrm{~nm}$ Au coating and $F_{\text {res }}=120 \mathrm{kHz}$ (NSG03/Au) for conductive measurements. Raman spectra was measured on microRaman spectrometer Centaur HR (Nanoscan Technology, Dolgoprudny, Russia) with a $100 \times$ objective at $532 \mathrm{~nm}$ (Cobolt, Solna, Sweden) with a beam spot of $\sim 1 \mu \mathrm{m}^{2}$ and laser power of $0.5 \mathrm{~mW}$.

\section{Results and Discussion}

Both BSA and BSA@GO solutions were stored in a refrigerator at $+4{ }^{\circ} \mathrm{C}$ and were stable within two weeks. The solutions were polymerized under UV light $\left(5 \mathrm{~W} / \mathrm{cm}^{2}\right.$ at $254 \mathrm{~nm}$ ) to prove the formation of polymer structures. To perform TPP, we first attached the $5 \mathrm{~mm}$ in diameter polydimethylsiloxane (PDMS) well to the glass cover slip. Following which, $30 \mu \mathrm{L}$ of solution was added to the well and placed under the objective of the optical microscope to supply femtosecond laser pulses with $25 \mathrm{~mW}$ power and scanning speed of $1 \mu \mathrm{m} / \mathrm{s}$. Note that there was not movement of the laser focused in $\mathrm{Z}$ axis during performing of TPP, and its variation is due to the non-planar substrate surface. We found that depending on the focus position of the laser that the nanowires of different diameters were formed (Figure 2). When laser focus is above the substrate, 3D polymerized "walls" grow perpendicular to the substrate surface (Figure 2a). The sample was washed, resulting in breaking down the "wall" and it parts falling onto a substrate (Figure 2b). We found that the "wall" consists of well-separated $30 \pm 2 \mu \mathrm{m}$ long nanowires with diameter of $320 \pm 70 \mathrm{~nm}$ (Figure 2c). The soft nature of hydrogel results in variations of measured diameter of nanowires. Nevertheless, the diameters of nanowires are well below the femtosecond laser wavelength as a result providing sub-wavelength lithography. When the laser focus is close to the substrate surface, the length and diameter of BSA hydrogel nanowires can be decreased to $20 \pm 2 \mu \mathrm{m}$ and $70 \pm 20 \mathrm{~nm}$, respectively (Figure $2 \mathrm{~d}-\mathrm{f}$ ).

The observed formation of TPP hydrogel nanowires can be explained in terms of the self-focusing effect of the laser beam due to the difference in liquid and polymerized hydrogel refraction indexes [21,22]. The effect provides an almost uniform cross-section of two-photon absorption over the whole filament length leading to high-aspect-ratio of the hydrogel nanowire (length/diameter $\approx 100$ ) as shown in Figure 2c. Formation of subwavelength nanostructures with period of $300 \pm 20 \mathrm{~nm}$ is well visible on the sidewalls of narrow nanowires (Figure $2 \mathrm{f}$, insert). We assume that the effect of self-trapped beams plays a major role in the formation of nanowires in BSA and MB solution, generating "walls" perpendicular to the substrate (Figure 2g). When a femtosecond laser beam is focused close to the glass surface, self-focusing leads to the activation and directional moving of PI, causing nanowire polymerization. For a smaller diameter, it is possible to resolve the PI waves along the laser beam with a period of $\lambda / 2$ leading to the periodical nanostructuring of the nanowire surface. After washing we observe the falling of "walls" resulting in directional nanowire positioning. The different positions of the fallen nanowires point out that these are individual isolated structures. The main bands in the Raman spectra (Figure $2 \mathrm{~h}$ ) of albumin were clearly observed [23]: $1338 \mathrm{~cm}^{-1}$ (deformation mode of $\mathrm{CH}$ bonds), $1452 \mathrm{~cm}^{-1}$ (deformation mode of $\mathrm{CH}_{2}$ bonds), and $1630 \mathrm{~cm}^{-1}$ (Amide I). The secondary structure of BSA when denaturized under ultrafast laser pulses changes its conformation causing Raman peaks shift [24]. An additional band of $1537 \mathrm{~cm}^{-1}$ responsible for $\mathrm{C}=\mathrm{C}$ stretching and shift in main peaks appeared due to protein photochemical polymerization. 

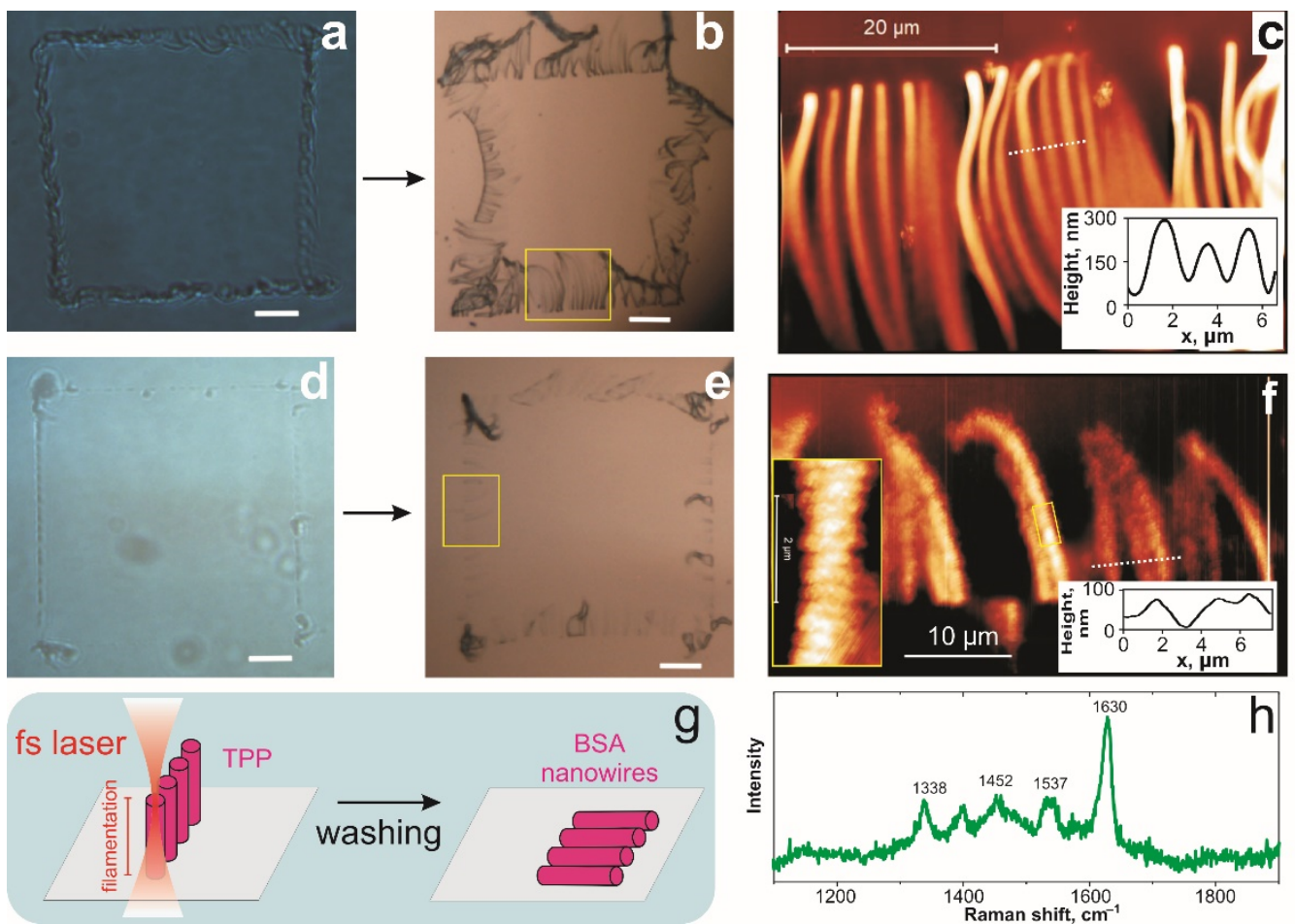

Figure 2. TPP of vertical BSA hydrogel nanowires: (a) Optical image of BSA hydrogel "walls" after polymerization under laser focused above the substrate; (b) optical image of "walls" after washing out non-polymerized BSA solution; (c) AFM image of "walls" marked by rectangular at (b). (d-f) The characterization of "walls" produced by laser focused close to substrate surface: optical image before (d) and after (e) washing; (f) AFM image of "walls" denoted by a rectangle of (e). The insert images on right (c, f): cross-section of individual nanowires. Left bottom insert of (f): enlarged AFM image of an individual nanowire with visible periodic sub-wavelength nanostructures; (g) scheme of BSA hydrogel nanowires fabrication by femtosecond pulsed laser; (h) Raman spectra of BSA hydrogel nanowires.

Some tasks of bio-inspired applications need precise and local positioning of individual nanowires. The direct laser writing (DLW) of hydrogels can be applied when moving substrate during the TPP process [25]. We performed DLW on both BSA and BSA@GO hydrogel nanowires on $\mathrm{Si} / \mathrm{SiO}_{2}$ substrate (Figure 3a). The TPP structures had become wider by up to $5 \mu \mathrm{m}$ with height of $100 \pm 20 \mathrm{~nm}$ (Figure 3b). SEM imaging reveals the low concentration of GO flakes that are randomly distributed in the polymerized matrix while pristine BSA have uniform structure (Figure 3c). The GO concentration is too low to be resolved by Raman spectroscopy in hydrogel. The shift in Raman spectra for Amide I band to $1626 \mathrm{~cm}^{-1}$ in BSA@GO hydrogel (Figure 3d) can be due to direct interaction of BSA and GO flakes during femtosecond laser processing [17] or stronger interaction of water molecules with defects in GO [26]. The interaction of albumin with GO leads to its partial unfolding that can be modulated by $\mathrm{pH}$ and ionic strength of solution [20,27]. It should be noted that during TPP there is possibility for reduction of GO resulting in increase of active oxygen groups [28,29]. In turn, it can stimulate the BSA polymerization close to graphene flakes. After the photochemical reduction of GO, the number of negative charges is decreased on the surfaces, which should decrease the repulsive energy of BSA [20] and hence increase the BSA on GO surface coverage.

The local electrical and mechanical properties of BSA and BSA@GO nanowires were investigated using atomic force microscopy with conductive cantilevers (for electrical measurement) and cantilevers with higher force constant (for nanoindentation characterization). For the former task we polymerized nanowires by DLW on gold substrate (Figure 4a,b). We observed good insulation properties of BSA@GO nanowires due to low concentration 
of GO in initial solution that prevents the formation of a percolating graphene network inside the BSA matrix. We discovered that there is an energy gap even between cantilever and gold surface contact. BSA molecules provide excellent properties of surfactant tending to form monolayer films on any hydrophobic surface (called "corona") [20]. Thus, the monolayer of non-polymerized BSA molecules on the gold surface can cause non-Ohmic contact even on flat gold surfaces.

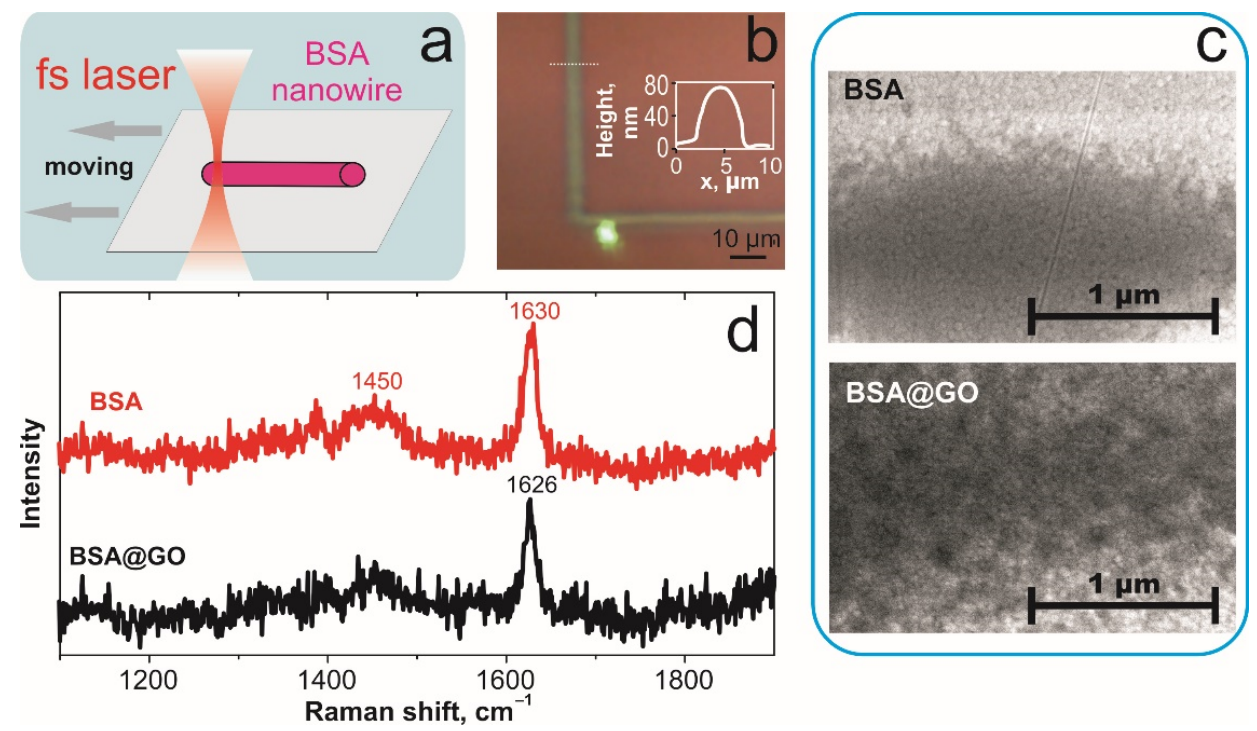

Figure 3. TPP of horizontal BSA hydrogel nanowires: (a) Scheme of DLW of BSA hydrogel nanowires on substrates; (b) optical image DLW BSA nanowires on silicon substrate. The insert image: cross section of DLW nanowire; (c) SEM image of BSA and BSA@GO hydrogel's structure; (d) Raman spectra of BSA and BSA@GO hydrogel nanowires.
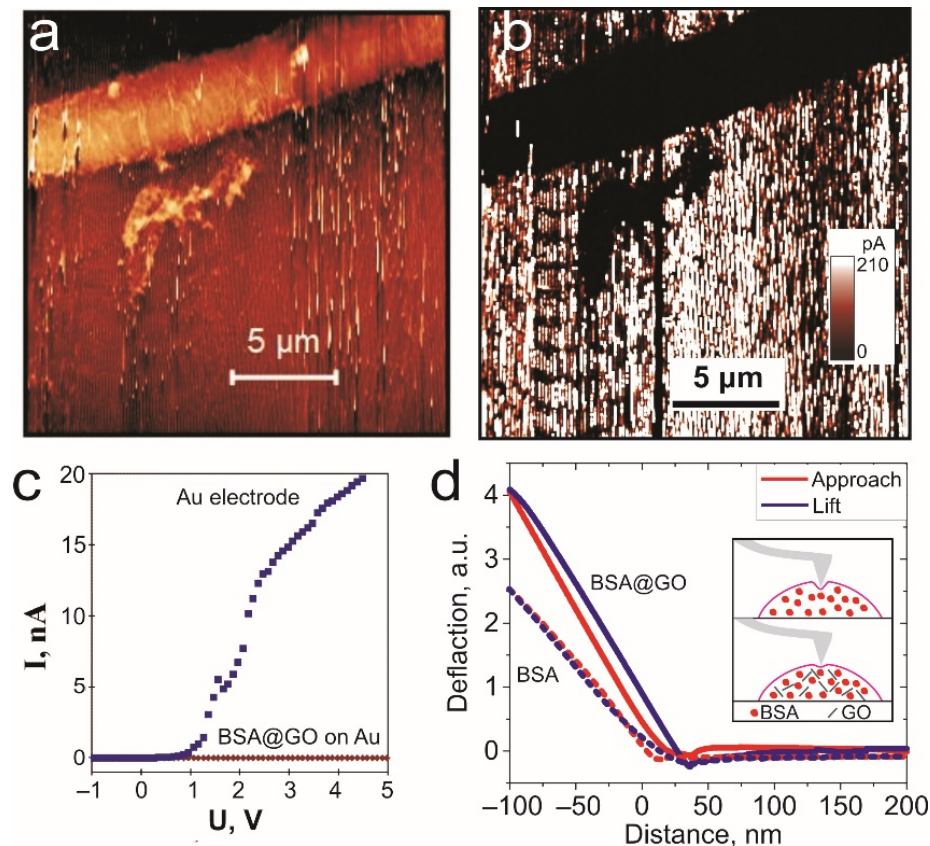

Figure 4. Local characterization of BSA@GO TPP nanowires: (a) AFM image of BSA@GO nanowire on gold substrate; (b) 2D map of spreading current of BSA@GO nanowire on gold. (c) Current vs. voltage spectra of contact between conductive AFM probe and BSA@GO nanowire and Au surface. (d) Deflection-distance curves of the BSA and BSA@GO nanowires. In the insert: schematics of the indentation of BSA and BSA@GO materials. 
Nanoscale mechanical properties of BSA@GO nanowires were investigated by local probing using standard silicon cantilevers. Effective Young's modulus of graphene oxide was estimated as $200 \mathrm{GPA}$ [30]. The addition of a small portion of GO to the BSA composite can greatly increase BSA@GO hardness. The cantilever deflection curves were used to estimate deflection depth and elastic properties (Table 1). The hardness is proportional to the maximal load on a deflection curve [31] and we can estimate the increase of hardness for BSA@GO compared to BSA from Figure 4d. The indentation depth decreases when the GO is added to the BSA hydrogel. We assume that the improvement of mechanical properties of BSA@GO hydrogel nanowires was due to rearrangement and structuring of BSA molecules during the polymerization close to the GO surface.

Table 1. The local mechanical properties of BSA and BSA@GO nanowires.

\begin{tabular}{ccc}
\hline Mechanical Parameters & BSA & BSA@GO \\
\hline Increase of hardness & 1 & 1.6 \\
Indentation depth & $35 \mathrm{~nm}$ & $20 \mathrm{~nm}$ \\
\hline
\end{tabular}

Increase in concentration of GO can both gradually improve the electrical properties and effect on mechanical properties of composite nanowires. Nevertheless, we have demonstrated that the gradual change in composite properties starts even for small concentrations of GO. Previously we demonstrated the biocompatibility of nanocomposites of BSA [14]. Nevertheless, additional investigation on cell proliferation has to be done for proper analysis of BSA@GO biocompatibility.

\section{Conclusions}

We have suggested the BSA and BSA@GO nanowires formation based on two-photon polymerization of protein photoactive solution. The novel route for nanofilaments growth with diameter down to $100 \mathrm{~nm}$ and aspect ratio up to 100 was demonstrated. The local electrical and mechanical properties of individual BSA@GO nanowires were investigated. The nanowires still provide good isolating properties for $0.005 \%$ GO concentration. Mechanical properties of nanowires were greatly improved for the BSA@GO composite. The hardness was increased up to 1.6 times. This suggested route provides novel bioinspired technology for functional biomimicking materials based on photoactive protein solutions and soluble graphene oxide.

Author Contributions: Conceptualization, A.Y.G. and V.N.; methodology, N.N. and I.B.; software, P.V.; validation, N.N., A.Y.G. and N.Y.; formal analysis, N.N.; investigation, N.N. and N.Y.; resources, A.Y.G. and I.B.; data curation, N.N.; writing—original draft preparation, N.N.; writing—review and editing, A.Y.G.; visualization, N.N.; supervision, V.N.; project administration, I.B.; funding acquisition, I.B. All authors have read and agreed to the published version of the manuscript.

Funding: The research was supported by the Russian Science Foundation under grant number 19-19-00401 (development and characterization of nanowires). I.B. participated in a project that received funding from the European Union's Horizon 2020 research and innovation programme under grant agreement $\mathrm{N}^{\circ} 739570$ (ANTARES).

Institutional Review Board Statement: Not applicable.

Informed Consent Statement: Not applicable.

Data Availability Statement: Not applicable.

Conflicts of Interest: The authors declare no conflict of interest. 


\section{References}

1. Otuka, A.J.G.; Tomazio, N.B.; Paula, K.T.; Mendonça, C.R. Two-Photon Polymerization: Functionalized Microstructures, MicroResonators, and Bio-Scaffolds. Polymers 2021, 13, 1994. [CrossRef] [PubMed]

2. Banerjee, H.; Suhail, M.; Ren, H. Hydrogel Actuators and Sensors for Biomedical Soft Robots: Brief Overview with Impending Challenges. Biomimetics 2018, 3, 15. [CrossRef] [PubMed]

3. Wu, L.; Hu, Y.; Tang, P.; Wang, H.; Bin, Y. High stretchable, pH-sensitive and self-adhesive rGO/CMCNa/PAA composite conductive hydrogel with good strain-sensing performance. Compos. Commun. 2021, 24, 100669. [CrossRef]

4. Cui, Z.; Zhou, M.; Greensmith, P.J.; Wang, W.; Hoyland, J.A.; Kinloch, I.A.; Freemont, T.; Saunders, B.R. A study of conductive hydrogel composites of pH-responsive microgels and carbon nanotubes. Soft Matter. 2016, 12, 4142-4153. [CrossRef]

5. Bobrinetskii, I.I.; Morozov, R.A.; Podgaetskii, V.M.; Simunin, M.M.; Yaminskii, I.V. A study of bulky nanotube composites based on albumin by high-resolution microscopy. Biophysics 2011, 56, 194-199. [CrossRef]

6. Simhon, D.; Gabay, I.; Shpolyansky, G.; Vasilyev, T.; Nur, I.; Meidler, R.; Hatoum, O.A.; Katzir, A.; Hashmonai, M.; Kopelman, D. Temperature-controlled laser-soldering system and its clinical application for bonding skin incisions. J. Biomed. Opt. 2015, 20, 128002. [CrossRef]

7. Zergioti, I.; Karaiskou, A.; Papazoglou, D.G.; Fotakis, C.; Kapsetaki, M.; Kafetzopoulos, D. Femtosecond laser microprinting of biomaterials. Appl. Phys. Lett. 2005, 86, 163902. [CrossRef]

8. Gerasimenko, A.Y.; Ten, G.N.; Ryabkin, D.I.; Shcherbakova, N.E.; Morozova, E.A.; Ichkitidze, L.P. The study of the interaction mechanism between bovine serum albumin and single-walled carbon nanotubes depending on their diameter and concentration in solid nanocomposites by vibrational spectroscopy. Spectrochim. Acta Part A Mol. Biomol. Spectrosc. 2020, 227, 117682. [CrossRef]

9. Wang, X.; Wei, Z.; Baysah, C.Z.; Zheng, M.; Xing, J. Biomaterial-based microstructures fabricated by two-photon polymerization microfabrication technology. RSC Adv. 2019, 9, 34472-34480. [CrossRef]

10. Ma, Z.-C.; Zhang, Y.-L.; Han, B.; Hu, X.-Y.; Li, C.-H.; Chen, Q.-D.; Sun, H.-B. Femtosecond laser programmed artificial musculoskeletal systems. Nat. Commun. 2020, 11, 4536. [CrossRef]

11. Fouassier, J.-P.; Morlet-Savary, F.; Lalevée, J.; Allonas, X.; Ley, C. Dyes as Photoinitiators or Photosensitizers of Polymerization Reactions. Materials 2010, 3, 5130-5142. [CrossRef]

12. Kaehr, B.; Shear, J.B. Multiphoton fabrication of chemically responsive protein hydrogels for microactuation. Proc. Natl. Acad. Sci. USA 2008, 105, 8850-8854. [CrossRef]

13. Lay, C.L.; Lee, Y.H.; Lee, M.R.; Phang, I.Y.; Ling, X.Y. Formulating an Ideal Protein Photoresist for Fabricating Dynamic Microstructures with High Aspect Ratios and Uniform Responsiveness. ACS Appl. Mater. Interfaces 2016, 8, 8145-8153. [CrossRef]

14. Markov, A.; Wördenweber, R.; Ichkitidze, L.; Gerasimenko, A.; Kurilova, U.; Suetina, I.; Mezentseva, M.; Offenhäusser, A.; Telyshev, D. Biocompatible SWCNT Conductive Composites for Biomedical Applications. Nanomaterials 2020, 10, 2492. [CrossRef]

15. Bobrinetskiy, I.I.; Seleznev, A.S.; Morozov, R.A.; Lopatina, O.A.; Podchernyaeva, R.Y.; Suetina, I.A. Investigation of the Effect of Local Electrical Stimulation on Cells Cultured on Conductive Single-Walled Carbon Nanotube/Albumin Films. J. Biomater. Nanobiotechnol. 2012, 3, 377-384. [CrossRef]

16. Ding, Z.; Ma, H.; Chen, Y. Interaction of graphene oxide with human serum albumin and its mechanism. RSC Adv. 2014, 4, 55290-55295. [CrossRef]

17. Nan, Z.; Hao, C.; Ye, X.; Feng, Y.; Sun, R. Interaction of graphene oxide with bovine serum albumin: A fluorescence quenching study. Spectrochim. Acta Part A Mol. Biomol. Spectrosc. 2019, 210, 348-354. [CrossRef]

18. Jokar, S.; Pourjavadi, A.; Adeli, M. Albumin-graphene oxide conjugates; carriers for anticancer drugs. RSC Adv. $2014,4,33001$. [CrossRef]

19. Joshi, S.; Singh, H.; Sharma, S.; Barman, P.; Saini, A.; Verma, G. Synthesis and characterization of graphene oxide-bovine serum albumin conjugate membrane for adsorptive removal of Cobalt(II) from water. Int. J. Environ. Sci. Technol. 2021, 18, 3915-3928. [CrossRef]

20. Liu, X.; Yan, C.; Chen, K.L. Adsorption of Human Serum Albumin on Graphene Oxide: Implications for Protein Corona Formation and Conformation. Environ. Sci. Technol. 2019, 53, 8631-8639. [CrossRef]

21. Biria, S.; Hosein, I.D. Control of Morphology in Polymer Blends through Light Self-Trapping: An in Situ Study of Structure Evolution, Reaction Kinetics, and Phase Separation. Macromolecules 2017, 50, 3617-3626. [CrossRef]

22. Jacobsen, A.J.; Barvosa-Carter, W.; Nutt, S. Micro-scale Truss Structures formed from Self-Propagating Photopolymer Waveguides. Adv. Mater. 2007, 19, 3892-3896. [CrossRef]

23. Lykina, A.; Artemyev, D.; Bratchenko, I. Analysis of albumin Raman scattering registration efficiency from different volume and shape cuvette. J. Biomed. Photonics Eng. 2017, 3, 020309. [CrossRef]

24. Murayama, K.; Tomida, M. Heat-Induced Secondary Structure and Conformation Change of Bovine Serum Albumin Investigated by Fourier Transform Infrared Spectroscopy. Biochemistry 2004, 43, 11526-11532. [CrossRef]

25. Yu, H.; Ding, H.; Zhang, Q.; Gu, Z.; Gu, M. Three-Dimensional Direct Laser Writing of PEGda Hydrogel Microstructures with Low Threshold Power using a Green Laser Beam. Light Adv. Manuf. 2021, 2, 1-8. [CrossRef]

26. Biscar, J.P.; Dhall, P.; Pennison, J. Raman behavior of bovine serum albumin. Chem. Phys. Lett. 1972, 14, 569-572. [CrossRef]

27. Taneva, S.G.; Krumova, S.; Bogár, F.; Kincses, A.; Stoichev, S.; Todinova, S.; Danailova, A.; Horváth, J.; Násztor, Z.; Kelemen, L.; et al. Insights into graphene oxide interaction with human serum albumin in isolated state and in blood plasma. Int. J. Biol. Macromol. 2021, 175, 19-29. [CrossRef] 
28. Li, X.; Zhang, Q.; Chen, X.; Gu, M. Giant refractive-index modulation by two-photon reduction of fluorescent graphene oxides for multimode optical recording. Sci. Rep. 2013, 3, 2819. [CrossRef]

29. Bobrinetskiy, I.I.; Emelianov, A.V.; Smagulova, S.A.; Komarov, I.A.; Otero, N.; Romero, P.M. Laser direct 3D patterning and reduction of graphene oxide film on polymer substrate. Mater. Lett. 2017, 187, 20-23. [CrossRef]

30. Zhou, J.; Cai, Q.; Xu, F. Nanoscale Mechanical Properties and Indentation Recovery of PI@GO Composites Measured Using AFM. Polymers 2018, 10, 1020. [CrossRef]

31. Oliver, W.C.; Pharr, G.M. An improved technique for determining hardness and elastic modulus using load and displacement sensing indentation experiments. J. Mater. Res. 1992, 7, 1564-1583. [CrossRef] 